\title{
Research on perfect binary correlation sequences based on higher-order cumulant
}

\author{
Yi Zhong ${ }^{*}$, Zheng Zhou and Ting Jiang
}

\begin{abstract}
Current researches define the perfect signal by the inner product of sequence itself and its delay sequence. However, these traditional sequences based on second-order statistics cannot handle colored Gaussian measurement noise automatically. Because higher-order cumulant (HOC) is insensitive to the adding Gaussian noise and symmetrical non-Gaussian noise, a new kind of perfect binary signal with good periodic correlation function based on HOC is presented. This paper proposes a new concept of perfect binary-third-order cyclic autocorrelation sequences (PBTOCAS) and defines the quasi-perfect binary-third-order cyclic autocorrelation sequences (QPBTOCAS) and the almost perfect binary-third-order cyclic autocorrelation sequences (APBTOCAS). Then the properties of these binary sequences are studied, and we theoretically prove that binary-third-order cyclic autocorrelation sequences can effectively suppress colored Gaussian noise. Finally, some QPBTOCAS and APBTOCAS with short lengths by computer searching are listed. From the observation of the PBTOCAS, we can see that it can well suit engineering applications, remedying the defect of the conventional pseudo-noise code used in very low signalnoise-ratio environments.
\end{abstract}

Keywords: Perfect signal, Higher-order cumulant, Correlation signal, Information theory

\section{Introduction}

Higher-order statistics (HOS) is a mathematical tool to describe the higher-order statistical properties of the random process, including higher-order cumulants and moments. Of course, the second-order cumulant is just the autocorrelation. A logical question to ask is "Why do we need higher-order cumulants; i.e., are not secondorder cumulants good enough?" Cumulants, therefore, not only display the amount of higher-order correlation, but also provide a measure of the distance of the random process from Gaussianity [1]. In fact, it has been shown that the estimation methods that exploit the nonGaussian signals have some inherent advantages over second-order method, such as (1) the ability to reconstruct the phase of non-minimum phase systems, (2) the array aperture is spread in space domain, and (3) the ability to separate non-Gaussian signals form Gaussian additive noise [2]. Based on the above characteristics, higher-order cumulant recently has been becoming a

\footnotetext{
* Correspondence: zhongyim2m@gmail.com

Key Laboratory of Universal Wireless Communication, Ministry of Education, Beijing University of Posts and Telecommunications, Beijing 100876, People's Republic of China
}

powerful tool for signal processing and system theory and widely used in many diverse fields; e.g., radar [3], sonar [4], telecommunication [1], oceanography [5], electromagnetism [6], geophysics [7], biomedical [8], and fault diagnosis [9]. The typical signal processing applications based on higher-order cumulant (HOC) mainly include the system identification and modeling of time series analysis [10], adaptive estimation and filtering [11], signal reconstruction [12], signal detection [13], harmonic retrieval [14], image processing [15], blind deconvolution and equalization [16], and array signal processing [17].

The main advantage of using high-order cumulant is that HOC is constantly zero for Gaussian process even in the colored Gaussian process. Harmonic retrieval, for example, uses the strong ability of HOC to suppress the Gaussian noise and harmonic signal, for flexible problem solving with signal parameter estimation in colored noise environments. Due to its great ability to overcome the noise interference, in this research, we suggest an attractive idea to expand the definition of perfect signal based on HOS.

\section{黑 Springer}


Perfect discrete signal and its design play key roles among many modern communication fields. The signals with good correlation properties have been widely applied to modem communication, such as radar, sonar, navigation, space ranging and controlling, and electronic antagonism systems. A perfect binary correlation signal is the best one whose periodic autocorrelation function is a single peak. However, the perfect binary sequence has been known only for a length of $N=4$ within sequence length $12,100[18,19]$.

Other kinds of perfect sequence are studied, such as perfect ternary sequences [20], perfect three-level and three-phase sequences [21], almost-perfect binary sequence [22], odd-periodic-perfect binary sequences [23], and Alexis sequences [24]. Current researches define the perfect signal by the inner product of sequence itself and its delay sequence. However, these traditional sequences based on second-order statistics cannot handle colored Gaussian measurement noise automatically, thus affecting the accuracy of their properties in engineering application. Since high-order cumulants are blind to any kind of Gaussian process, this paper introduces $\mathrm{HOC}$ into the field of perfect binary sequence, which breaks through the limitations of the perfect signal defined by second-order statistics, to fill out the blank of perfect signal in the area of research on Gaussian noise suppression.

In this paper, we propose a new concept of perfect binary sequence based on higher-order cumulant, which is the perfect binary-third-order cyclic autocorrelation sequence. The detailed analysis proves that the proposed sequences improve the performance of anti-noise for perfect signal significantly, and more perfect signals could be obtained in engineering application with this method. The rest of this paper is organized as follows: In Section 2, we present the definition of the proposed sequences. In Section 3, we study the properties of the sequences. In Section 4, we apply the above properties to search out some quasi-perfect binary-third-order cyclic autocorrelation sequences (QPBTOCAS) and almost perfect binary-third-order cyclic autocorrelation sequences (APBTOCAS) with short lengths by computer, and the periodic correlation performances of the sequences are analyzed by MATLAB (MathWorks, Natick, MA, USA). In Section 5, we conclude our work and discuss future research.

\section{Definitions}

Definition 1 Suppose a sequence $x(n)=\left(x_{0}, x_{1}, \cdots, x_{N-1}\right)$ with length $N, x(n)$ is defined as the binary sequence, if $x(n) \in\{-1,1\}$ holds.

Definition 2 The imbalance of sequence $x(n)$ with length $N$ is defined to be

$$
I=\sum_{n=0}^{N-1} x(n)=n_{p}-n_{q}
$$

where $n_{p}$ and $n_{q}$ respectively denote the numbers of 1 and -1 in the binary sequence $x(n)$.

Definition 3 Suppose a sequence $x(n)=\left(x_{0}, x_{1}, \cdots, x_{N}\right.$ $\left.{ }_{1}\right)$ with length $N$ and its imbalance satisfies $I \in\{-1,0,1\}$, $x(n)$ is considered as a zero-mean or a closely approximate zero-mean stationary random process, then the third-order cumulant of cyclic autocorrelation binary sequence $x(n)$ is defined as

$$
c_{3 x}\left(\tau_{1}, \tau_{2}\right)=\frac{1}{N} \sum_{n=1}^{N} x(n) x\left(n+\tau_{1}\right) x\left(n+\tau_{2}\right)
$$

where $0 \leq \tau_{1} \leq N-1,0 \leq \tau_{2} \leq N-1$, and $E$ denotes the maximum value.

Definition 4 Suppose a binary sequence $x(n)=\left(x_{0}, x_{1}\right.$, ..., $\left.x_{N-1}\right)$ with length $N$ and its imbalance satisfies $I \in$ $\{-1,0,1\}$, then $x(n)$ is defined as the perfect binary-third -order cyclic autocorrelation sequences (PBTOCAS) if there exists a value $\tau_{1}=u(0 \leq u \leq N-1)$ and satisfies the following equation:

$$
c_{3 x}\left(u, \tau_{2}\right)= \begin{cases}E & \tau_{2}=m \\ 0 & \tau_{2} \neq m\end{cases}
$$

where $0 \leq m \leq N-1$, and $E$ denotes the maximum value.

Definition 5 Suppose a random binary sequence $x(n)$ $=\left(x_{0}, x_{1}, \ldots, x_{N-1}\right)$ with length $N$ and its imbalance satisfies $I \in\{-1,0,1\}$, then $x(n)$ is defined as the QPBTOCAS, if there exists a value $\tau_{1}=u(0 \leq u \leq N-1)$ and satisfies the following equation:

$$
\hat{c}_{3 x}\left(u, \tau_{2}\right)= \begin{cases}E & \tau_{2}=m \\ \mathrm{a} & \tau_{2} \neq m\end{cases}
$$

where $0 \leq m \leq N-1, a \rightarrow 0, a \ll E$, and $E$ denotes the maximum value.

Definition 6 Suppose a binary sequence $x(n)=\left(x_{0}, x_{1}\right.$, ..., $\left.x_{N-1}\right)$ with length $N$ and its imbalance satisfies $I \in$ $\{-1,0,1\}$, then $x(n)$ is defined as the APBTOCAS if there exists a value $\tau_{1}=u(0 \leq u \leq N-1)$ and satisfies the following equation:

$$
c_{3 x}\left(u, \tau_{2}\right)=\left\{\begin{array}{ll}
E & \tau_{2}=m \\
-E & \tau_{2}=n \\
0 & \tau_{2} \neq m, n
\end{array},\right.
$$

where $0 \leq m \leq N-1,0 \leq n \leq N-1$, and $E$ denotes the maximum value. 
Definition 7 The imbalance $I$ of a perfect binarythird-order cyclic autocorrelation sequence with length $N$ is given by

$$
I=0
$$

The sequence is defined to be a constant-weight PBTOCAS, otherwise defined as a non-constant-weight PBTOCAS.

On the other hand, we have similar definition to the QPBTOCAS and APBTOCAS.

\section{Transformation features}

It will take a huge amount of time to do an exhaustive search for the PBTOCAS, QPBTOCAS, and APBTO CAS in length $N$. According to the definition in Section 2 and the important properties of higher-order cumulant , we can get some features as follows to reduce the search domain significantly. Since these sequences all belong to the class of binary-third-order sequence, we take PBTOCAS as example to prove the common transformation feature.

If sequence $x(n)=\left(x_{0}, x_{1}, \ldots, x_{N-1}\right)$ is PBTOCAS with length $N$, we can get the following four important properties.

Proposition 1 Reverse transformation. If PBTOCAS $x$ (n) after the transformation $x_{1}(n)=-x(n)$, then sequence $x_{1}(n)$ is PBTOCAS.

Proof It is easy to verify the propositions mentioned above by using the definition of PBTOCAS in (3), so we leave them out here.

Proposition 2 Mapping transformation. If PBTOCAS $x(n)$ after the transformation $x_{1}(n)=x(-n)$, then sequence $x_{1}(n)$ is PBTOCAS.

Proof Consider the sequence $x(n)=\left(x_{0}, x_{1}, \ldots, x_{N-1}\right)$ with length $N$, where $c_{3 x}\left(\tau_{1}, \tau_{2}\right)$ is the third-order cumulant defined in (3). We have

$$
\begin{aligned}
c_{3 x_{1}}\left(\tau_{1}, \tau_{2}\right)= & \frac{1}{N} \sum_{n=0}^{N-1} x_{1}(n) x_{1}\left(n+\tau_{1}\right) x_{1}\left(n+\tau_{2}\right) \\
= & \frac{1}{N}\left\{x_{1}(0) x_{1}\left(\tau_{1}\right) x_{1}\left(\tau_{2}\right)+\cdots+x_{1}(N-1) x_{1}\right. \\
& \left.\left(N-1+\tau_{1}\right) x_{1}\left(N-1+\tau_{2}\right)\right\} \\
& =\frac{1}{N}\left\{x(0) x\left(\tau_{1}\right) x\left(\tau_{2}\right)+x(-1) x\left(-1+\tau_{1}\right) x\left(-1+\tau_{2}\right)\right. \\
& \left.+\cdots+x(-N+1) x\left(-N+1+\tau_{1}\right) x\left(-N+1+\tau_{2}\right)\right\} \\
& =\frac{1}{N}\left\{x(N) x\left(N+\tau_{1}\right) x\left(N+\tau_{2}\right)+x(N-1) x\right. \\
& \left.\left(N-1+\tau_{1}\right) x\left(N-1+\tau_{2}\right)+\cdots+x(1) x\left(1+\tau_{1}\right) x\left(1+\tau_{2}\right)\right\} \\
& =\frac{1}{N}\left\{x(0) x\left(\tau_{1}\right) x\left(\tau_{2}\right)+\cdots+x(N-1) x\left(N-1+\tau_{1}\right) x\left(N-1+\tau_{2}\right)\right\} \\
& =c_{3 x}\left(\tau_{1}, \tau_{2}\right)
\end{aligned}
$$

According to the definition in (3), we prove that sequence $x_{1}(n)$ is PBTOCAS. This completes the proof.
Proposition 3 Reverse order transformation. If PBTO CAS $x(n)$ after the transformation $x_{1}(n)=x(N-n)$, then sequence $x_{1}(n)$ is PBTOCAS.

Proof Consider the sequence $x(n)=\left(x_{0}, x_{1}, \ldots, x_{N-1}\right)$ with length $N$, where $c_{3 x}\left(\tau_{1}, \tau_{2}\right)$ is the third-order cumulant defined in (3). We have

$$
\begin{aligned}
c_{3 x_{1}}\left(\tau_{1}, \tau_{2}\right)= & \frac{1}{N} \sum_{n=0}^{N-1} x_{1}(n) x_{1}\left(n+\tau_{1}\right) x_{1}\left(n+\tau_{2}\right) \\
= & \frac{1}{N} \sum_{n=0}^{N-1} x(N-n) x\left(N-n-\tau_{1}\right) x\left(N-n-\tau_{2}\right) \\
= & \frac{1}{N}\left\{x(N) x_{1}\left(N-\tau_{1}\right) x_{1}\left(N-\tau_{2}\right)+x_{1}(N-1) x_{1}\right. \\
& \left.\left(N-1+\tau_{1}\right) x_{1}\left(N-1+\tau_{2}\right)+\cdots+x(1) x_{1}\left(1-\tau_{1}\right) x_{1}\left(1-\tau_{2}\right)\right\} \\
& =\frac{1}{N}\left\{x(0) x\left(0-\tau_{1}\right) x\left(0-\tau_{2}\right)+\cdots+x(N-1) x\left(N-1-\tau_{1}\right) x\right. \\
& \left.\left(N-1-\tau_{2}\right)\right\} \\
& =c_{3 x}\left(N-\tau_{1}, N-\tau_{2}\right)
\end{aligned}
$$

According to the definition in (3), we prove that sequence $x_{1}(n)$ is a PBTOCAS. This completes the proof.

Proposition 4 Cyclic shifting transformation. If PBTO CAS $x(n)$ after the transformation $x_{1}(n)=x(n+u), u \leq N$, then sequence $x_{1}(n)$ is PBTOCAS.

Proof Consider the sequence $x_{1}(n)=\left(x_{0}, x_{1}, \ldots, x_{N-1}\right)$ with length $N$, where $c_{3 x}\left(\tau_{1}, \tau_{2}\right)$ is the third-order cumulant defined in (3). We have

$$
\begin{aligned}
c_{3 x_{1}}\left(\tau_{1}, \tau_{2}\right) & =\frac{1}{N} \sum_{n=0}^{N-1} x_{1}(n) x_{1}\left(n+\tau_{1}\right) x_{1}\left(n+\tau_{2}\right) \\
& =\frac{1}{N} \sum_{n=0}^{N-1} x(n+u) x\left(n+u+\tau_{1}\right) x\left(n+u+\tau_{2}\right) \\
& =\frac{1}{N} \sum_{n=u}^{N-1+u} x(n) x\left(n+\tau_{1}\right) x\left(n+\tau_{2}\right) .
\end{aligned}
$$

Since $n+\tau_{1}=\left(n+\tau_{1}\right) \bmod N, \quad n+\tau_{2}=\left(n+\tau_{2}\right) \bmod N$, hence,

$$
\begin{aligned}
c_{3 x_{1}}\left(\tau_{1}, \tau_{2}\right) & =\frac{1}{N} \sum_{n=u}^{N-1+u} x(n) x\left(n+\tau_{1}\right) x\left(n+\tau_{2}\right) \\
& =\hat{c}_{3 x}\left(\tau_{1}, \tau_{2}\right) .
\end{aligned}
$$

According to the definition in (3), we prove that sequence $x_{1}(n)$ is PBTOCAS. This completes the proof.

The proof of the propositions mentioned of QPBTO CAS and APBTOCAS is similar to the PBTOCAS, so we leave it out here.

\section{Searching results}

We use the propositions 1 to 4 in Section 3 to reduce the searching range effectively, since all the QPBTOCAS and the APBTOCAS seem only one if 
Table 1 Some searching results of QPBTOCAS within length 25

\begin{tabular}{lll}
\hline Length & Sequence (octal) & Position of $\boldsymbol{\tau}_{\mathbf{1}}=\boldsymbol{u}$ \\
\hline 7 & 113 & $0,1,2,3,4,5$ \\
\cline { 2 - 3 } & 116 & $0,1,2,3,4,5$ \\
\hline 15 & 4657 & $1,2,3,4,5,6,7 \cdots 14$ \\
\cline { 2 - 3 } & 7531 & $1,2,3,4,5,6,7 \cdots 14$ \\
\hline
\end{tabular}

The sequence is denoted as octal, where ' 1 ' means ' +1 ' and ' 0 ' means ' -1 '.

they can get from one sequence by using the propositions 1 to 4 . Therefore, by means of an exhaustive computer search for the possible class of PBTOCAS, we get some QPBTOCAS within length 25 in Table 1 and APBTOCAS within length 26 listed in Table 2.

\subsection{Some searching results of QPBTOCAS}

For example, a sequence 116 (octal) with length 7 denotes the sequence $x(n)=\{-1,-1,-1,-1,-1,+1,+1,-$ $1,+1,+1,-1,+1,+1,+1\}$, then sequence $x(n)$ is QPBTOCAS.

From the output of the third-order cumulant $c_{3 x}$ $\left(\tau_{1}, \tau_{2}\right)$ of the 4657 (octal) sequence, we can find that there are 14 peak values and we can see below the contour and the mesh plotting for the sequence, and it is clear that it has peak points with value $=-1$, and any other value will be $= \pm 1 / 15 \rightarrow 0$. The results are shown in Figures 1 and 2, represented in mesh and contour plots. At the $\left(\tau_{1}, \tau_{2}\right)$ pair, the fork indicates that there is a peak value.

Among the 14 peaks, we choose 14 sets of peaks in Table 3. In each set, we can see that only one peak will be produced in the same value with the change of shift count $\tau_{2}$, and any other value will be 0 when the another shift count $\tau_{1}=$ $1,2,3,4,5,6,7 \cdots 14$, which are the same as the theoretical results.

As a result, when we fix one shift count $\tau_{1}$ in Table 1 and move another shift count $\tau_{2}$ of the sequence, we can get one set of the peak location $\left(\tau_{1}, \tau_{2}\right)$ in Table 3 while the value at any other point will be equal to nearly 0 , which indicates that the 4657 (octal) QPBTOCAS has a good periodic correlation performance.

\subsection{Some searching results of APBTOCAS}

For example, a sequence 667 (octal) with length 14 denotes the sequence $x(n)=\{-1,-1,-1,-1,-1,+1,+$

Table 2 Some searching results of APBTOCAS within length 26

\begin{tabular}{lll}
\hline Length & Sequence (octal) & Position of $\boldsymbol{\tau}_{\mathbf{1}}=\boldsymbol{u}$ \\
\hline 14 & 667 & $2,4,5,6,8,9,10,12$ \\
\hline
\end{tabular}

The sequence is denoted as octal, where ' 1 ' means ' +1 ' and ' 0 ' means ' -1 '.

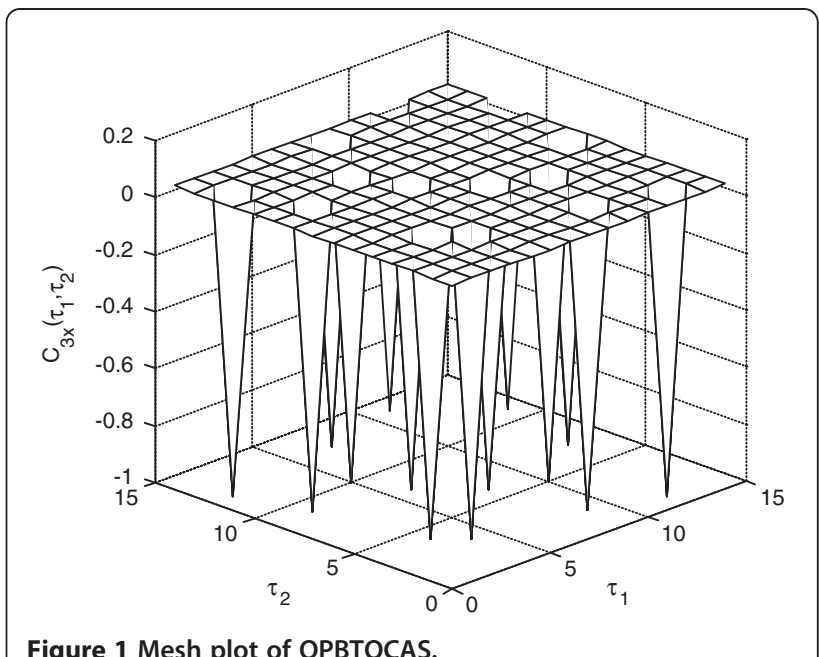

Figure 1 Mesh plot of QPBTOCAS.

$1,-1,+1,+1,-1,+1,+1,+1\}$ the sequence $x(n)$ is an APBTOCAS.

From the output of the third-order cumulant $c_{3 x}$ $\left(\tau_{1}, \tau_{2}\right)$ of the 667 (octal)sequence, we can find that there are 36 peak values and we can see below the contour and the mesh plotting for the sequence, and it is clear that it has peak points with value = $\pm 8 / 15 \approx 0.5333$, and any other value will be equal to 0 . The results are shown in Figures 3 and 4, represented in mesh and contour plots. At the $\left(\tau_{1}, \tau_{2}\right)$ pair, the circle indicates that there is a positive peak, and the fork indicates that there is a negative peak.

Among the 36 peaks, we choose eight sets of peaks in Table 4. In each set, we can see that two

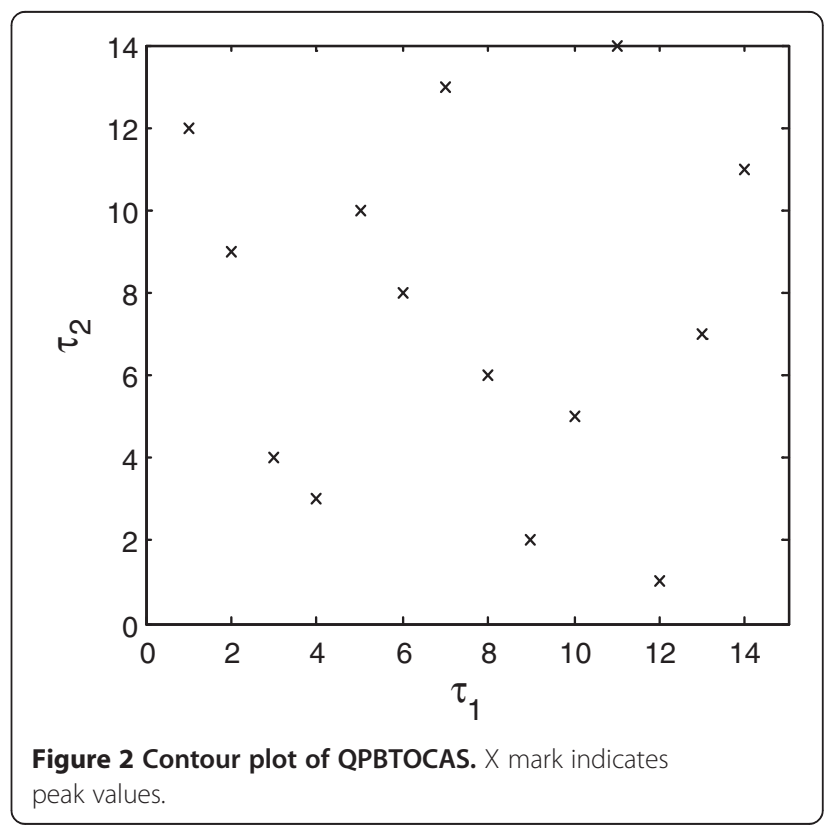


Table 3 Peaks locations for sequence 667 (octal)

\begin{tabular}{lllllllllllllll}
\hline & \multicolumn{11}{c}{ Set } \\
\cline { 2 - 15 } & $\mathbf{1}$ & $\mathbf{2}$ & $\mathbf{3}$ & $\mathbf{4}$ & $\mathbf{5}$ & $\mathbf{6}$ & $\mathbf{7}$ & $\mathbf{8}$ & $\mathbf{9}$ & $\mathbf{1 0}$ & $\mathbf{1 1}$ & $\mathbf{1 2}$ & $\mathbf{1 3}$ & $\mathbf{1 4}$ \\
\hline$\tau_{1}$ & 1 & 2 & 3 & 4 & 5 & 6 & 7 & 8 & 9 & 10 & 11 & 12 & 13 & 14 \\
\hline$\tau_{2}$ & 12 & 9 & 4 & 3 & 10 & 8 & 13 & 6 & 2 & 5 & 14 & 1 & 7 & 11
\end{tabular}

opposite peaks will be produced in the same value with the change of shift count $\tau_{2}$ and any other value will be 0 , when another shift count is $\tau_{1}=$ $2,4,5,6,8,9,10,12$, which are the same as the theoretical results. As a result, when we fix one shift count $\tau_{1}$ in Table 2 and move another shift count $\tau_{2}$ of the sequence, we can get one set of the peak location $\left(\tau_{1}, \tau_{2}\right)$ in Table 4 while the value at any other point will be equal to 0 , which indicates that the 667 (octal) APBTOCAS has a good periodic correlation performance.

\section{Conclusions}

A new concept of PBTOCAS is proposed in the paper. Since the higher-order cumulant is more robust to additive measurement noise than correlation, even if that noise is colored, this article introduces higher-order cumulants into the research of perfect binary sequence, which can enrich the research field of perfect signal theory. According to the properties of HOC, the PBTOCAS can draw itself out of Gaussian noise, thereby boosting their signal-to-noise ratios in engineering application.

Because of the limit of program algorithm and the computer operating speed, only some QPBTOCAS and APBTOCAS have been found. Although we have not found the PBTOCAS of the definition in (3) at present, the realization of QPBTOCAS and

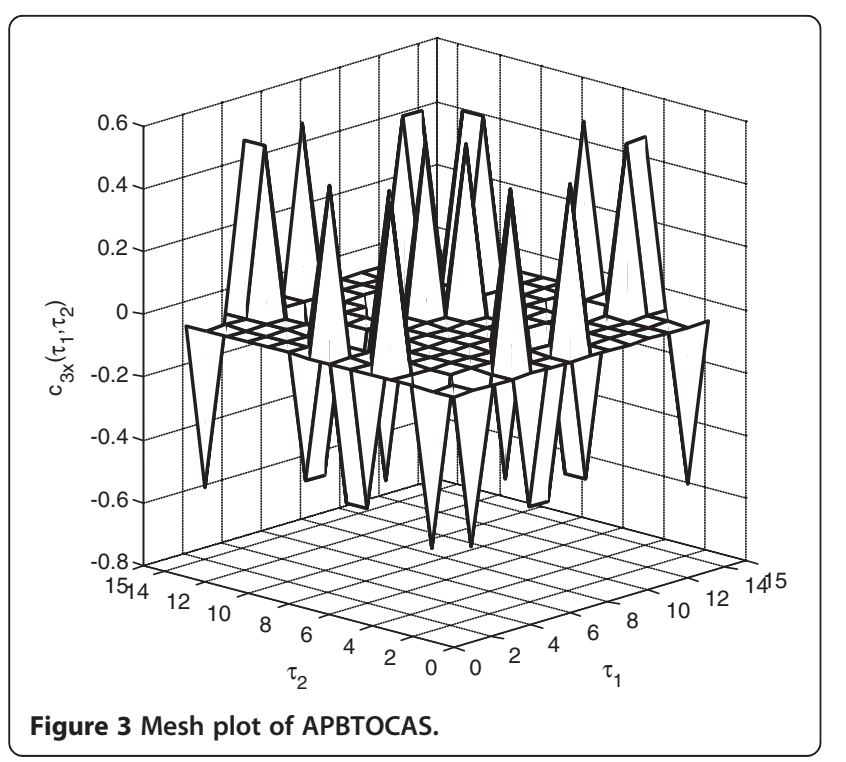

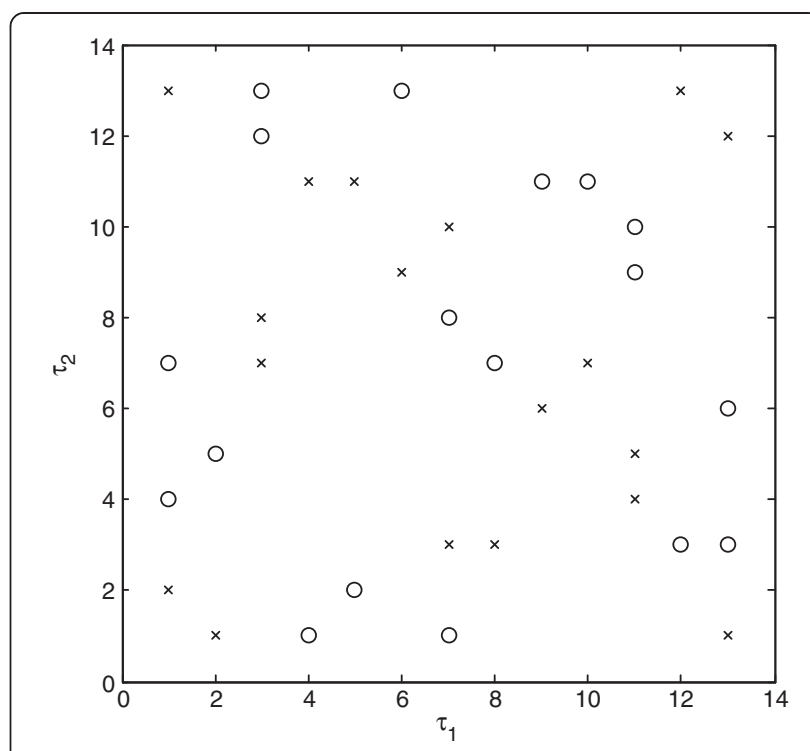

Figure 4 Contour plot of APBTOCAS. The circle mark indicates positive peaks, and the $x$ mark indicates negative peaks.

APBTOCAS could be further constructed according to the conjecture. On the other hand, the existence of the PBTOCAS also requires further study. For the binary sequence within length 25 whose imbalance holds, searching result shows that these sequences are all QPBTOCAS. Then for the binary sequence within length 26 whose imbalance holds, searching result shows that these sequences are all almost PBTOCAS. Based on the above results, we can draw a conclusion that there exist the nonconstant-weight QPBTOCAS and the constantweight APBTOCAS, and the number of QPBTOCAS is larger than that of APBTOCAS. At present, although all QPBTOCAS are displayed as $\mathrm{M}$ sequences by computer searching, it is essential to prove whether all $M$ sequences conform to the definition in (4) in further research. Furthermore, other possibility of the existence of the QPBTOCAS needs to be verified in the further computer search. Meanwhile, the APBTOCAS with good cyclic autocorrelation function shown in Table 2 can be widely used in the engineering application with a strict requirement on bit error rate.

Table 4 Peaks locations for sequence 667 (octal)

\begin{tabular}{cccccccccccccccc}
\hline Set $\mathbf{1}$ & Set $\mathbf{2}$ & Set $\mathbf{3}$ & \multicolumn{2}{c}{ Set $\mathbf{4}$} & Set $\mathbf{5}$ & Set $\mathbf{6}$ & \multicolumn{2}{c}{ Set $\mathbf{7}$} & \multicolumn{2}{c}{ Set $\mathbf{8}$} \\
\hline $\boldsymbol{\tau}_{\mathbf{1}}$ & $\boldsymbol{\tau}_{\mathbf{2}}$ & $\boldsymbol{\tau}_{\mathbf{1}}$ & $\boldsymbol{\tau}_{\mathbf{2}}$ & $\boldsymbol{\tau}_{\mathbf{1}}$ & $\boldsymbol{\tau}_{\mathbf{2}}$ & $\boldsymbol{\tau}_{\mathbf{1}}$ & $\boldsymbol{\tau}_{\mathbf{2}}$ & $\boldsymbol{\tau}_{\mathbf{1}}$ & $\boldsymbol{\tau}_{\mathbf{2}}$ & $\boldsymbol{\tau}_{\mathbf{1}}$ & $\boldsymbol{\tau}_{\mathbf{2}}$ & $\boldsymbol{\tau}_{\mathbf{1}}$ & $\boldsymbol{\tau}_{\mathbf{2}}$ & $\boldsymbol{\tau}_{\mathbf{1}}$ & $\boldsymbol{\tau}_{\mathbf{2}}$ \\
\hline 2 & 1 & 4 & 1 & 5 & 2 & 6 & 9 & 8 & 3 & 9 & 6 & 10 & 7 & 12 & 3 \\
\hline 2 & 5 & 4 & 11 & 5 & 11 & 6 & 13 & 8 & 7 & 9 & 11 & 10 & 11 & 12 & 13 \\
\hline
\end{tabular}




\section{Competing interests}

The authors declare that they have no competing interests.

\section{Acknowledgments}

This article is a revised and expanded version of a paper entitled 'Research on theory of almost perfect binary-third-order autocorrelation sequences' presented at IEEE International Conference on

Communications, Signal Processing and Systems, Beijing China last 17 October 2012

This work was supported by Important National Science and Technology Specific Projects (2010ZX03006-006), NSFC (61171176), Scientific Research Fund of Zhejiang Provincial Education Department under grant no. Y201225956 and Natural Science Foundation of Ningbo under grant no. 2012A610015.

Received: 9 February 2013 Accepted: 11 April 2013

Published: 9 May 2013

\section{References}

1. JM Mendel, Tutorial on higher-order statistics (spectra) in signal processing and system theory: theoretical results and some applications. Proc. IEEE 79(3), 278-305 (1991)

2. $L$ Feng, $L$ Jingqing, $L$ Shaoqian, Signal direction of arrival estimation based high-order cumulants and a partially calibrated array, in International Conference on Communications, Circuits and Systems, Xiamen University, Fujian Province, 25-28 June 2006, (IEEE Press. Piscataway 1, 293-297 (2006)

3. VK Anandan, R Ramachandra, Spectral analysis of atmospheric radar signal using higher order spectral estimation technique. IEEE Trans. Geoscience and Remote Sensing 39(9), 1890-1895 (2001)

4. A Trucco, Experimental results on the detection of embedded objects by a prewhitening filter. IEEE J. Ocean. Eng. 26(4), 783-794 (2001)

5. A Bendjama, S Bourennane, Localization of buried objects through higher order statistics techniques, in 2003 IEEE Workshop on Statistical Signal Processing, ed. by (, , ). 28 September to 1 October 2003 (IEEE Press, Piscataway, 2003) pp. 474-477

6. X Yougen, L Zhiwen, KT Wong, C Jinliang, Virtual-manifold ambiguity in HOS-based direction-finding with electromagnetic vector-sensors. IEEE Trans. Aerospace and Electronic Systems 44(4), 1291-1308 (2008)

7. GL Lazear, Mixed-phase wavelet estimation using fourth order cumulants. Geophysic 58(7), 1042-1051 (1993)

8. S Osowski, LT Hoai, T Markiewicz, Support vector machine-based expert system for reliable heartbeat recognition. IEEE Trans. Biomedical Engineering 51(4), 582-589 (2004)

9. LAI Wu-xing, PW Tse, Z Gui-cai, Classification of gear faults using cumulants and the radial basis function network. Mech. Syst. Signal Process. 18(2), 381-389 (2004)

10. A Swami, JM Mendel, Identifiability of the AR parameter estimaters of an ARMA process using cumulants. IEEE Trans. Automatic Control 37(2), 268-273 (1992)

11. D Aboutajdine, A Adib, A Meziane, Fast adaptive algorithms for AR parameters estimation using higher order statistics. IEEE Trans. Signal Process. 44(8), 1998-2009 (1996)

12. AP Petropulu, CL Nikias, Blind convolution using signal reconstruction from partial higher order cepstral information. IEEE Trans. Signal Process. 41(6), 2088-2095 (1993)

13. BM Sadler, GB Giannakis, L Keh-Shin, Estimation and detection in non-Gaussian noise using higher order statistics. IEEE Trans. Signal Process. 42(10), 2729-2741 (1994)

14. S Zhenghao, FW Fairman, Harmonic retrieval via state space and fourth-order cumulants. IEEE Trans. Signal Processing 42(5), 1109-1119 (1994)

15. H Farid, Blind inverse gamma correction. IEEE Trans. Image Process. 10(10), 1428-1433 (2001)

16. F Chih-Chun, C Chong-Yung, Performance of cumulant based inverse filters for blind deconvolution. IEEE Trans. Signal Process. 47(7), 1922-1935 (1999)

17. P Chevalier, A Ferreol, On the virtual array concept for the fourth-order direction finding problem. IEEE Trans. Signal Process. 47(9), 2592-2595 (1999)

18. YY Xian, Existence of one dimensional perfect binary arrays. Electron. Lett. 23(24), 1277-1278 (1987)

19. L Bomer, M Antweiler, Perfect energy efficient sequences. Electron. Lett. 27(15), 1332-1334 (1991)

20. TH Hold, J Justesen, Ternary sequences with perfect periodic autocorrelation. IEEE Trans. Inform. Theory IT 29(4), 597-600 (1983)
21. L Bomer, M Antweiler, Perfect three-level and three-phase sequence and arrays. IEEE Trans. Comm. 42(2/3/4), 767-772 (1994)

22. P Langevin, Almost perfect binary functions. App. Alg. Eng. Comm. Comp. 4, 95-102 (1993)

23. L Anon, Mismatch filtering of odd-periodic binary sequences. IEEE Trans. Aero. Elec. Syst 34(4), 1345-1350 (1998)

24. HD Luke, Binary Alexis sequences with perfect correlation. IEEE Trans. Commun. 49(6), 966-968 (2001)

doi:10.1186/1687-1499-2013-123

Cite this article as: Zhong et al:: Research on perfect binary correlation sequences based on higher-order cumulant. EURASIP Journal on Wireless Communications and Networking 2013 2013:123.

\section{Submit your manuscript to a SpringerOpen ${ }^{\odot}$ journal and benefit from:}

- Convenient online submission

Rigorous peer review

- Immediate publication on acceptance

- Open access: articles freely available online

- High visibility within the field

- Retaining the copyright to your article

Submit your next manuscript at $>$ springeropen.com 